UPR-971-T

\title{
Gauge Symmmetry and Supersymmetry Breaking by Discrete Symmetry
}

\author{
Tianjun Li t \\ Department of Physics and Astronomy, University of Pennsylvania, \\ Philadelphia, PA 19104
}

\begin{abstract}
We study the principles of the gauge symmetry and supersymmetry breaking due to the local or global discrete symmetries on the extra space manifold. We show that the gauge symmetry breaking by Wilson line is the special case of the discrete symmetry approach where all the discrete symmetries are global and act freely on the extra space manifold. As applications, we discuss the $N=2$ supersymmetric $S O(10)$ and $E_{8}$ breaking on the space-time $M^{4} \times A^{2}$ and $M^{4} \times D^{2}$, and point out that similarly one can study any $N=2$ supersymmetric $S O(M)$ breaking. We also comment on the one-loop effective potential, the possible questions and generalization.
\end{abstract}

PACS: 11.25.Mj; 11.10.Kk; 04.65.+e; 11.30.Pb

Keywords: Discrete Symmetry; Symmetry Breaking; Extra Dimensions

December 2001

\footnotetext{
${ }^{1}$ E-mail: tli@bokchoy.hep.upenn.edu, phone: (215) 573-5820, fax: (215) 898-2010.
} 


\section{Introduction}

Grand Unified Theory (GUT) gives us an simple and elegant understanding of the quantum numbers of quarks and leptons, and the success of gauge coupling unification in the Minimal Supersymmetric Standard Model strongly supports this idea. Right now, the Grand Unified Theory at high energy scale has been widely accepted, however, there are some problems in GUT: the grand unified gauge symmetry breaking mechanism, the doublet-triplet splitting problem, and the proton decay problem, etc.

Recently, a new scenario proposed to address above questions in GUT has been discussed extensively [1, 2, 3, 4. The key point is that the supersymmetric GUT model exists in 5 or higher dimensions and is broken down to the 4-dimensional $N=1$ supersymmetric Standard like Model for the zero modes due to the discrete symmetries in the neighborhoods of the branes or on the extra space manifolds, which become non-trivial constraints on the multiplets and gauge generators in GUT [3]. The attractive models have been constructed explicitly, where the supersymmetric 5-dimensional and 6-dimensional GUT models are broken down to the 4-dimensional $N=1$ supersymmetric $S U(3) \times S U(2) \times U(1)^{n-3}$ model, where $n$ is the rank of GUT group, through the compactification on various orbifolds and manifolds. The GUT gauge symmetry breaking and doublet-triplet splitting problems have been solved neatly by the discrete symmetry projections. Other interesting phenomenology, like $\mu$ problems, gauge coupling unifications, non-supersymmetric GUT, gauge-Higgs unification, proton decay, etc, have also been discussed [1, 2, 3, 团.

In this paper, we discuss the gauge symmetry and supersymmetry breaking by the local or global discrete symmetries on the extra space manifold in general. We prove the theorems for the gauge symmetry on the fixed point, line or hypersurface, and in the bulk for the zero modes. And we explain why the high dimensional supersymmetry is broken down to the 4-dimensional $N=1$ supersymmetry. These general results form the foundation of this approach, which we would like to call it the discrete symmetry approach. We also show that the gauge symmetry breaking by Wilson line is the special case of the discrete symmetry approach, where all the discrete symmetries are global and act freely on the extra space manifold.

In the previous discussions [1, 3], the $S O(10)$ is broken by the Klein discrete symmetries $Z_{2} \times Z_{2}$ on the extra space manifold. So, we would like to discuss the $S O(10)$ breaking by one cyclic discrete symmetry. In addition, although from the phenomenological point of view $E_{8}$ is not very interesting, we would like to discuss the $E_{8}$ breaking because of technical interesting. In short, we study the $N=2$ supersymmetric $S O(10)$ breaking and $E_{8}$ breaking on the space-time $M^{4} \times A^{2}$ and $M^{4} \times D^{2}$, where $M^{4}$ is the 4-dimensional Minkowski space-time, $A^{2}$ is the 2-dimensional annulus, and $D^{2}$ is the 2-dimensional disc. We list all the constraints on constructing the 4-dimensional $N=1$ supersymmetric $S U(3) \times S U(2) \times U(1)^{n-3}$ model for the zero modes, in which $n$ is the rank, $i$. e., $n=5$ and 8 for $S O(10)$ and $E_{8}$, respectively. Moreover, we point out that on the space-time $M^{4} \times A^{2}$ and $M^{4} \times D^{2}$, we can break any $N=2$ supersymmetric $S O(M)$ models down to the 4-dimensional $N=1$ supersymmetric $S U(3) \times S U(2) \times U(1)^{n-3}$ models for the zero modes in which $n$ is the rank 
of the group $S O(M)$. Furthermore, we comment on the one-loop effective potential, possible questions and generalization in the discrete symmetry approach.

We would like to explain our convention. For simplicity, we define the $n \times n$ diagonal matrix as $\left(\alpha_{1}, \alpha_{2}, \ldots, \alpha_{n}\right)$, for example, we define the $3 \times 3$ diagonal matrix as

$$
\left(\alpha_{1}, \alpha_{2}, \alpha_{3}\right) \equiv\left(\begin{array}{ccc}
\alpha_{1} & 0 & 0 \\
0 & \alpha_{2} & 0 \\
0 & 0 & \alpha_{3}
\end{array}\right) .
$$

In addition, suppose $G$ is a Lie group and $H$ is a subgoup of $G$. In general, for $G=$ $S U(N)$ and $G=S O(N), H$ can be the subgroup of $U(N)$ and $O(N)$, respectively. We denote the commutant of $H$ in $G$ as $G / H, i$. e.,

$$
G / H \equiv\{g \in G \mid g h=h g, \text { for any } h \in H\}
$$

And if $H_{1}$ and $H_{2}$ are the subgroups of $G$, we define

$$
G /\left\{H_{1} \cup H_{2}\right\} \equiv\left\{G / H_{1}\right\} \cap\left\{G / H_{2}\right\}
$$

\section{Gauge Symmmetry and Supersymmetry Break- ing by Discrete Symmetry}

We assume that in a $(4+\mathrm{n})$-dimensional space-time manifold $M^{4} \times E$ where $M^{4}$ is the 4-dimensional Minkowski space-time and $E$ is the extra n-dimensional space manifold, there exist some local or global discrete symmetries on the extra space manifold and the Lagrangian is invariant under the local or global discrete symmetries. Moreover, the discrete symmetries may not act freely on $E$. When the discrete symmetry does not act freely on $E$, there exists a brane at each fixed point, line or hypersurface, where the Standard Model fermions can be located.

Suppose we have $K$ discrete symmetries, and each discrete symmetry forms a cyclic group $\Gamma_{J}$, for $J=1,2, \ldots K$. Let us use the $I-t h$ discrete symmetry as a representative for discussion, which forms the cyclic group $\Gamma_{I}$. As we know, the local discrete symmetry is defined in the special brane's open neighborhood, where the position of the special brane is the only fixed point, line or hypersurface as long as the open neighborhood is small enough [2, 3]. In the open neighborhood of the special brane, the local discrete symmetry is global. So, if the $I-t h$ discrete symmetry is local, we can concentrate on the open neighborhood of the special brane. In short, without loss of generality, we can assume that the $I-t h$ discrete symmetry is global for simplicity.

Assume the coordinates for the extra dimensions are $y^{1}, y^{2}, \ldots, y^{n}$, the action of any element $\gamma_{i}^{I} \subset \Gamma_{I}$ on $E$ can be expressed as

$$
\gamma_{i}^{I}: \quad\left(y^{1}, y^{2}, \ldots, y^{n}\right) \longrightarrow\left(\gamma_{i}^{I} y^{1}, \gamma_{i}^{I} y^{2}, \ldots, \gamma_{i}^{I} y^{n}\right)
$$



$\gamma_{i}^{I} \subset \Gamma_{I}$

The Lagrangian is invariant under the discrete symmetry, i. e., for any element

$$
\mathcal{L}\left(x^{\mu}, \gamma_{i}^{I} y^{1}, \gamma_{i}^{I} y^{2}, \ldots, \gamma_{i}^{I} y^{n}\right)=\mathcal{L}\left(x^{\mu}, y^{1}, y^{2}, \ldots, y^{n}\right)
$$

So, for a generic bulk multiplet $\Phi$ which fills a representation of the bulk gauge group $G$, we have

$$
\Phi\left(x^{\mu}, \gamma_{i}^{I} y^{1}, \gamma_{i}^{I} y^{2}, \ldots, \gamma_{i}^{I} y^{n}\right)=\eta_{\Phi}^{I}\left(R_{\gamma_{i}^{I}}\right)^{l_{\Phi}} \Phi\left(x^{\mu}, y^{1}, y^{2}, \ldots, y^{n}\right)\left(R_{\gamma_{i}^{I}}^{-1}\right)^{m_{\Phi}}
$$

where $\eta_{\Phi}^{I}$ can be determined from the Lagrangian (up to $\Gamma_{I}$ for the matter fields), $l_{\Phi}$ and $m_{\Phi}$ are the non-negative integers determined by the representation of $\Phi$ under the bulk gauge group $G$. In general, $\eta_{\Phi}^{I}$ is an element in $\Gamma_{I}$, for example $\Gamma_{I}=Z_{2}$, $\eta_{\Phi}^{I}= \pm 1$. Moreover, $R_{\gamma_{i}^{I}}$ is an element in $G$, and $R_{\Gamma_{I}}$ is a discrete subgroup of $G$. We will choose $R_{\gamma_{i}^{I}}$ as the matrix representation for $\gamma_{i}^{I}$ in the adjoint representation of the gauge group $G$. The consistent condition for $R_{\gamma_{i}^{I}}$ is

$$
R_{\gamma_{i}^{I}} R_{\gamma_{j}^{I}}=R_{\gamma_{i}^{I} \gamma_{j}^{I}}, \forall \gamma_{i}^{I}, \gamma_{j}^{I} \subset \Gamma_{I}
$$

Mathematical speaking, the map $R: \Gamma_{I} \longrightarrow R_{\Gamma_{I}} \subset G$ is a homomorphism.

Furthermore, suppose $\Gamma_{I}=Z_{N}$, for a generic field $\phi$ in $\Phi$, we have

$$
\phi\left(x^{\mu}, \gamma_{i}^{I} y^{1}, \gamma_{i}^{I} y^{2}, \ldots, \gamma_{i}^{I} y^{n}\right)=\omega^{\kappa_{i}^{I}(\phi)} \phi\left(x^{\mu}, y^{1}, y^{2}, \ldots, y^{n}\right)
$$

where

$$
\omega=e^{\mathrm{i} \frac{2 \pi}{N}}
$$

and $\kappa_{i}^{I}(\phi)$ is an integer which can be determined from Eq. (6). And if $\kappa_{i}^{I}(\phi)=0 \bmod$ $N, \phi$ has zero mode.

Theorem I. For the zero modes, the gauge symmetry is $G /\left\{R_{\Gamma_{1}} \cup R_{\Gamma_{2}} \cup \ldots \cup\right.$ $\left.R_{\Gamma_{K}}\right\}$.

Proof. We first consider the $I-t h$ discrete symmetry and assume that $\gamma_{i}^{I}$ is the generator of the cyclic group $\Gamma_{I}$. For the gauge field $A=A_{\mu}^{a} T^{a}, \eta_{A}^{I}=0$. So, for a fixed field $A_{\mu}^{a} T^{a}$, we have

$$
A_{\mu}^{a}\left(x^{\mu}, \gamma_{i}^{I} y^{1}, \gamma_{i}^{I} y^{2}, \ldots, \gamma_{i}^{I} y^{n}\right) T^{a}=A_{\mu}^{a}\left(x^{\mu}, y^{1}, y^{2}, \ldots, y^{n}\right) R_{\gamma_{i}^{I}} T^{a}\left(R_{\gamma_{i}^{I}}^{-1}\right)
$$

In addition, if $A_{\mu}^{a} T^{a}$ has zero mode, it satisfies the equation

$$
A_{\mu}^{a}\left(x^{\mu}, \gamma_{i}^{I} y^{1}, \gamma_{i}^{I} y^{2}, \ldots, \gamma_{i}^{I} y^{n}\right) T^{a}=A_{\mu}^{a}\left(x^{\mu}, y^{1}, y^{2}, \ldots, y^{n}\right) T^{a}
$$


Then, we obtain that $A_{\mu}^{a} T^{a}$ has zero mode only if $\left[R_{\gamma_{i}^{I}}, T^{a}\right]=0, i . e ., R_{\gamma_{i}^{I}}$ and $T^{a}$ are commute. So, under the discrete symmetry $\Gamma_{I}$, the gauge symmetry is $G / R_{\Gamma_{I}}$ for the zero mode. Because we have the same results for the other discrete symmetries, for the zero modes, the gauge symmetry is $G /\left\{R_{\Gamma_{1}} \cup R_{\Gamma_{2}} \cup \ldots \cup R_{\Gamma_{K}}\right\}$. The theorem is proved.

Theorem II. If $\left(y^{1}=u^{1}, y^{2}=u^{2}, \ldots, y^{n}=u^{n}\right)$ is the fixed point of $\Gamma_{I}$ and is not the fixed point of the rest discrete symmetries, the gauge symmetry at that fixed point is $G / R_{\Gamma_{I}}$.

Proof. Suppose $\gamma_{i}^{I}$ is the generator of the cyclic group $\Gamma_{I}$. At the fixed point $\left(y^{1}=u^{1}, y^{2}=u^{2}, \ldots, y^{n}=u^{n}\right)$ of $\Gamma_{I}$, we have

$$
A_{\mu}^{a}\left(x^{\mu}, \gamma_{i}^{I} u^{1}, \gamma_{i}^{I} u^{2}, \ldots, \gamma_{i}^{I} u^{n}\right) T^{a}=A_{\mu}^{a}\left(x^{\mu}, u^{1}, u^{2}, \ldots, u^{n}\right) T^{a}
$$

Similar to the proof of Theorem I, we obtain that the gauge symmetry at that fixed point is $G / R_{\Gamma_{I}}$. We would like to emphasize that the gauge symmetry $G / R_{\Gamma_{I}}$ is preserved for all the KK modes.

Corollary I. Suppose $H$ is the subgroup of $\Gamma_{I}$, at the fixed point of $H$ which is not the fixed point of $\Gamma_{I}$ and the rest discrete symmetries, the gauge symmetry is $G / R_{H}$ where $R_{H} \subseteq R_{\Gamma_{I}}$.

Corollary II. If $\Gamma_{I}$ and $\Gamma_{J}$ have common fixed point which is not the fixed point of the rest discrete symmetries, the gauge symmetry at that common fixed point is $G /\left\{R_{\Gamma_{I}} \cup R_{\Gamma_{J}}\right\}$.

Corollary III. Suppose $S \subset E$ is the fixed hypersurface of $\Gamma_{I}$ and is not fixed under the rest discrete symmetries, the gauge symmetry on $S$ is $G / R_{\Gamma_{I}}$. In addition, if $S$ is the common fixed hypersurface of $\Gamma_{I}$ and $\Gamma_{J}$, and $S$ is not fixed under the rest discrete symmetries, the gauge symmetry on $S$ is $G /\left\{R_{\Gamma_{I}} \cup R_{\Gamma_{J}}\right\}$.

Supersymmetry Breaking. High dimensional supersymmetry corresponds to the 4-dimensional $N>1$ supersymmetry. In terms of the 4-dimensional $N=$ 1 supersymmetry language, the gauge multiplet can be decomposed into a vector multiplet $V$ and the chiral multiplets $\Phi_{1}, \Phi_{2}, \ldots, \Phi_{2 k+1}$ in the adjoint representation. Under the discrete symmetries, $\partial_{y^{i}}$ or $\partial_{z^{i}}$ in the complex coordinate may not be invariant, for example, under the reflection $Z_{2}$ symmetry on the $i$ - th coordinate $y^{i}$, $\partial_{y^{i}} \rightarrow-\partial_{y^{i}}$. Then, under the discrete symmetry, the transformations of the chiral mutlipets $\Phi_{1}, \Phi_{2}, \ldots, \Phi_{2 k+1}$ may be different from the transformation of the vector 
multiplet $V$. Therefore, if all the component fields of the chiral multiplets $\Phi_{1}, \Phi_{2}, \ldots$, $\Phi_{2 k+1}$ did not have the zero modes, we have the 4-dimensional $N=1$ supersymmetry for the zero modes. Similarly, one can discuss the 4-dimensional supersymmetry on the fixed point, lines or hypersuface. By the way, we might keep the zero modes of some fields in the chiral multiplets, which can be considered as $S U(2)_{L}$ Higgs doublets in some models.

Relation to the Wilson Line Approach. Assume that $\Gamma_{I}$ is a global symmetry and acts freely on the extra space manifold $E$, we can define the quotient manifold $B=E / \Gamma_{I}$. Because $\Gamma_{I}$ belongs to the fundamental group of $B, i$. e.,

$$
\Gamma_{I} \subseteq \pi_{1}(B)
$$

the discrete symmetry approach become the Wilson line approach for $\Gamma_{I}$ [5, 6], $i . e$, the gauge symmetry breaking by Wilson line.

Furthermore, if all the discrete symmetries are global and act freely on the extra space manifold $E$, we can define the quotient manifold $B=E /\left(\Pi_{J=1}^{K} \Gamma_{J}\right)$. And then, $\Pi_{J=1}^{K} \Gamma_{J}$ belong to the fundamental group of $B, i$. e.,

$$
\Pi_{J=1}^{K} \Gamma_{J} \subseteq \pi_{1}(B)
$$

Therefore, the Wilson line approach is the special case of the discrete symmetry approach where all the discrete symmetries are global and act freely on the extra space manifold.

To be explicit, let us give an example. Consider the 5-dimensional $N=1$ supersymmetric GUT models on the space-time $M^{4} \times S^{1}$, we can not break the 4dimensional $N=2$ supersymmetry down to $N=1$ supersymmetry in the Wilson line approach. However, one can do this in the discrete symmetry approach by considering the refelction symmetries [1, 2]. And if we moduloed the refelction symmetries, the fundamental group of the extra space orbifold is trivial, , $i$. e., $\pi_{1}\left(S^{1} / Z_{2}\right)$ or $\pi_{1}\left(S^{1} /\left(Z_{2} \times Z_{2}^{\prime}\right)\right)$ is trivial.

\section{$3 \quad N=2$ Supersymmetric Theory on $M^{4} \times A^{2}$ and $M^{4} \times D^{2}$}

In this section, we would like to review the $N=2$ supersymmetric theory on the space-time $M^{4} \times A^{2}$ and $M^{4} \times D^{2}$, where $A^{2}$ and $D^{2}$ are the 2-dimensional annulus and disc, respectively.

The convenient coordinates for the annulus $A^{2}$ is polar coordinates $(r, \theta)$, and it is easy to change them to the complex coordinates by $z=r e^{\mathrm{i} \theta}$. We call the innner radius of the annulus as $R_{1}$, and the outer radius of the annulus as $R_{2}$. When $R_{1}=0$, the annulus becomes the disc $D^{2}$, which is an special case of $A^{2}$. We can define the 
$Z_{n}$ symmetry on the annulus $A^{2}$ by the equivalent class

$$
z \sim \omega z,
$$

where $\omega=e^{\mathrm{i} \frac{2 \pi}{n}}$. And we denote the corresponding generator for $Z_{n}$ as $\Omega$ which satifies $\Omega^{n}=1$. The KK mode expansions and the detail of this set-up can be found in Ref. [3].

The $N=2$ supersymmetry in 6-dimension has 16 supercharges and corresponds to the $N=4$ supersymmetry in 4 -dimension, thus, only the gauge multiplet can be introduced in the bulk. This multiplet can be decomposed under the 4-dimensional $N=1$ supersymmetry into a vector multiplet $V$ and three chiral multiplets $\Sigma, \Phi$, and $\Phi^{c}$ in the adjoint representation, with the fifth and sixth components of the gauge field, $A_{5}$ and $A_{6}$, contained in the lowest component of $\Sigma$. The Standard Model fermions are on the boundary 4-brane at $r=R_{1}$ or $r=R_{2}$ for the annulus $A^{2}$ scenario, and on the 3-brane at origin or on the boundary 4-brane at $r=R_{2}$ for the disc $D^{2}$ scenario.

In the Wess-Zumino gauge and 4-dimensional $N=1$ supersymmetry language, the bulk action is [7]

$$
\begin{aligned}
S= & \int d^{6} x\left\{\operatorname{Tr}\left[\int d^{2} \theta\left(\frac{1}{4 k g^{2}} \mathcal{W}^{\alpha} \mathcal{W}_{\alpha}+\frac{1}{k g^{2}}\left(\Phi^{c} \partial \Phi-\frac{1}{\sqrt{2}} \Sigma\left[\Phi, \Phi^{c}\right]\right)\right)+\text { h.c. }\right]\right. \\
& +\int d^{4} \theta \frac{1}{k g^{2}} \operatorname{Tr}\left[\left(\sqrt{2} \partial^{\dagger}+\Sigma^{\dagger}\right) e^{-V}(-\sqrt{2} \partial+\Sigma) e^{V}\right] \\
& \left.+\int d^{4} \theta \frac{1}{k g^{2}} \operatorname{Tr}\left[\Phi^{\dagger} e^{-V} \Phi e^{V}+\Phi^{c \dagger} e^{-V} \Phi^{c} e^{V}\right]\right\} .
\end{aligned}
$$

From above action, we obtain the transformations of gauge multiplet under $\Omega$ as

$$
\begin{gathered}
V\left(\omega z, \omega^{n-1} \bar{z}\right)=\left(R_{\Omega}\right)^{l_{V}} V(z, \bar{z})\left(R_{\Omega}^{-1}\right)^{m_{V}}, \\
\Sigma\left(\omega z, \omega^{n-1} \bar{z}\right)=\omega^{n-1}\left(R_{\Omega}\right)^{l_{\Sigma}} \Sigma(z, \bar{z})\left(R_{\Omega}^{-1}\right)^{m_{\Sigma}}, \\
\Phi\left(\omega z, \omega^{n-1} \bar{z}\right)=\omega^{n-1}\left(R_{\Omega}\right)^{l_{\Phi}} \Phi(z, \bar{z})\left(R_{\Omega}^{-1}\right)^{m_{\Phi}}, \\
\Phi^{c}\left(\omega z, \omega^{n-1} \bar{z}\right)=\omega^{2}\left(R_{\Omega}\right)^{l_{\Phi c}} \Phi^{c}(z, \bar{z})\left(R_{\Omega}^{-1}\right)^{m_{\Phi c}},
\end{gathered}
$$

where $\left(l_{V}, m_{V}\right),\left(l_{\Sigma}, m_{\Sigma}\right),\left(l_{\Phi}, m_{\Phi}\right)$ and $\left(l_{\Phi^{c}}, m_{\Phi^{c}}\right)$ are determined from the representation under the gauge group. Because we will decompose the bulk GUT group $G$ into its maximal subgroup, which is the product of several groups, for example $G \supset G_{1} \times G_{2} \times G_{k}$, we define $\left(l_{V}, m_{V}\right)$ as $\left(l_{V}^{1}, m_{V}^{1}\right) \otimes\left(l_{V}^{2}, m_{V}^{2}\right) \otimes \ldots \otimes\left(l_{V}^{k}, m_{V}^{k}\right)$, where $\left(l_{V}^{i}, m_{V}^{i}\right)$ is determined from the decomposed representation of $V$ under $G_{i}$. Similar notation will be used for $\left(l_{\Sigma}, m_{\Sigma}\right),\left(l_{\Phi}, m_{\Phi}\right)$ and $\left(l_{\Phi^{c}}, m_{\Phi^{c}}\right)$. To be explicit, we will give $\left(l_{V}, m_{V}\right),\left(l_{\Sigma}, m_{\Sigma}\right),\left(l_{\Phi}, m_{\Phi}\right)$ and $\left(l_{\Phi^{c}}, m_{\Phi^{c}}\right)$ in detail in the following discussions. 


\section{$4 S O(10)$ Breaking on $M^{4} \times A^{2}$ and $M^{4} \times D^{2}$}

In the previous discussions [1], 3], the $S O(10)$ is broken by the Klein discrete symmetries $Z_{2} \times Z_{2}$ on the extra space manifold. So, we would like to discuss the $S O(10)$ breaking by one cyclic discrete symmetry on the space-time $M^{4} \times A^{2}$ and $M^{4} \times D^{2}$. We will break the 6-dimensional $N=2$ supersymmetric $S O(10)$ model down to 4dimensional $N=1$ supersymmetric $S U(3) \times S U(2) \times U(1)^{2}$ model for the zero modes. Because $S O(10) \supset S U(5) \times U(1), S U(4) \times S U(2) \times S U(2)$ and $S O(8) \times U(1)$ [8], and we can not obtain the Standard Model from $S O(8) \times U(1)$, we decompose the $S O(10)$ into $S U(5) \times U(1)$ and $S U(4) \times S U(2) \times S U(2)$ in the following discussions of $S O(10)$ breaking.

\section{$4.1 \quad$ Model I: $S O(10) \supset S U(5) \times U(1)$}

In this subsection, we discuss the $S O(10)$ breaking by considering its maximal subgroup $S U(5) \times U(1)$.

The gauge fields of $S O(10)$ are in the adjoint representation of $S O(10)$ with dimension 45. Under the gauge group $S U(5) \times U(1)$, the $S O(10)$ gauge fields decompose as 8

$$
45=(24,0) \oplus(\mathbf{1 0}, 4) \oplus(\overline{\mathbf{1 0}},-4) \oplus(\mathbf{0}, \mathbf{1})
$$

Assume that we have $Z_{n}$ symmetry on $A^{2}$ or $D^{2}$ and $\Omega$ is a generator of $\Gamma=Z_{n}$, we choose the following matrix representation for $\Omega$, which will give us the representations of all the elements in $\Gamma$,

$$
R_{\Omega}=\left(\omega^{n_{1}}, \omega^{n_{1}}, \omega^{n_{1}}, \omega^{n_{2}}, \omega^{n_{2}}\right) \otimes(+1)
$$

where $\omega=e^{\mathrm{i} \frac{2 \pi}{n}}$. Therefore, we only need to concentrate on $S U(5)$. In addition, $\left(l_{V}, m_{V}\right),\left(l_{\Sigma}, m_{\Sigma}\right),\left(l_{\Phi}, m_{\Phi}\right)$ and $\left(l_{\Phi^{c}}, m_{\Phi^{c}}\right)$ are equal to $(1,1)$ if the gauge field were in the representation $(\mathbf{2 4}, \mathbf{0})$, and $\left(l_{V}, m_{V}\right),\left(l_{\Sigma}, m_{\Sigma}\right),\left(l_{\Phi}, m_{\Phi}\right)$ and $\left(l_{\Phi^{c}}, m_{\Phi^{c}}\right)$ are equal to $(2,0)$ if the gauge fields were in the representation $(\mathbf{1 0}, \mathbf{4})$, and $\left(l_{V}, m_{V}\right),\left(l_{\Sigma}, m_{\Sigma}\right)$, $\left(l_{\Phi}, m_{\Phi}\right)$ and $\left(l_{\Phi^{c}}, m_{\Phi^{c}}\right)$ are equal to $(0,2)$ if the gauge fields were in the representation $(\overline{10},-4)$.

In order to have the models with $S U(3) \times S U(2) \times U(1)^{2}$ gauge symmetry and 4-dimensional $N=1$ supersymmetry for the zero modes, we obtain the following constraints on $n_{i}$

(a) $3 n_{1}+2 n_{2}=0 \bmod n$,

(b) $n_{1} \neq n_{2} \bmod n$,

$$
\text { (c) }\left|n_{1}-n_{2}\right| \neq 1 \text { and } n-2 \bmod n \text {, }
$$




$$
\text { (d) }\left|n_{i}+n_{j}\right| \neq 0,1 \text { and } n-2 \bmod n, \text { for } i, j=1,2 \text {. }
$$

Because $R_{\Omega} \subset S U(5) \times U(1)$, we obtain the constraint (a). And the constraint (b) will break the $S U(5)$ down to $S U(3) \times S U(2) \times U(1)$. In addition, the constraint (c) will project out all the zero modes of $\Sigma, \Phi$ and $\Phi^{c}$ in the representation $(\mathbf{2 4}, \mathbf{0})$, and the constraint (e) will project out all the zero modes of $V, \Sigma, \Phi$ and $\Phi^{c}$ in the representations $(\mathbf{1 0}, \mathbf{4})$ and $(\overline{\mathbf{1 0}}, \mathbf{- 4})$. By the way, the zero modes of $\Sigma, \Phi$ and $\Phi^{c}$ in the representation $(\mathbf{0}, \mathbf{1})$ are automatically projected out.

Let us give the model with $Z_{16}$ symmetry, the matrix representation for $R_{\Omega}$ is

$$
R_{\Omega}=\left(\omega^{2}, \omega^{2}, \omega^{2}, \omega^{5}, \omega^{5}\right) \otimes(+1) .
$$

It is easy to check that all the constraints are satisfied.

First, we consider that the extra space manifold is the annulus $A^{2}$. For the zero modes, we have 4-dimensional $N=1$ supersymmetry and $S U(3) \times S U(2) \times U(1)^{2}$ gauge symmetry in the bulk and on the 4-branes at $r=R_{1}$ and $r=R_{2}$. Including the KK states, we will have the 4-dimensional $N=4$ supersymmetry and $S O(10)$ gauge symmetry in the bulk, and on the 4-branes at $r=R_{1}$ and $r=R_{2}$.

Second, we consider that the extra space manifold is the disc $D^{2}$. For the zero modes, we have 4-dimensional $N=1$ supersymmetry and $S U(3) \times S U(2) \times U(1)^{2}$ gauge symmetry in the bulk and on the 4-brane at $r=R_{2}$. Including all the KK states, we will have the 4-dimensional $N=4$ supersymmetry and $S O(10)$ gauge symmetry in the bulk, and on the 4-brane at $r=R_{2}$. In addition, because the origin $(r=0)$ is the fixed point under the $Z_{16}$ symmetry, we always have the 4-dimensional $N=1$ supersymmetry and $S U(3) \times S U(2) \times U(1)^{2}$ gauge symmetry on the 3 -brane at origin in which only the zero modes exist. And if we put the Standard Model fermions on the 3-brane at origin, the extra dimensions can be large and the gauge hierarchy problem can be solved for there does not exist the proton decay problem at all.

\section{$4.2 \quad$ Model II: $S O(10) \supset S U(4) \times S U(2) \times S U(2)$}

In this subsection, we discuss the $S O(10)$ breaking by considering its maximal subgroup $S U(4) \times S U(2) \times S U(2)$.

The gauge fields of $S O(10)$ are in the adjoint representation of $S O(10)$ with dimension 45. Under the gauge group $S U(4) \times S U(2)_{L} \times S U(2)_{R}$, the $S O(10)$ gauge fields decompose as 8

$$
45=(15,1,1) \oplus(1,3,1) \oplus(1,1,3) \oplus(6,2,2) .
$$

Assume that we have $Z_{n}$ symmetry on $A^{2}$ or $D^{2}$ and $\Omega$ is a generator of $\Gamma=Z_{n}$, we choose the following matrix representation for $\Omega$, which will give us the representations of all the elements in $\Gamma$,

$$
R_{\Omega}=\left(\omega^{n_{1}}, \omega^{n_{1}}, \omega^{n_{1}}, \omega^{n_{2}}\right) \otimes(+1,+1) \otimes\left(\omega^{n_{3}}, \omega^{n_{4}}\right)
$$


where $\omega=e^{\mathrm{i} \frac{2 \pi}{n}}$. Moreover, $\left(l_{V}, m_{V}\right),\left(l_{\Sigma}, m_{\Sigma}\right),\left(l_{\Phi}, m_{\Phi}\right)$ and $\left(l_{\Phi^{c}}, m_{\Phi^{c}}\right)$ are equal to $(1,1) \otimes(0,0) \otimes(0,0)$ if the gauge fields were in the representation $(\mathbf{1 5}, \mathbf{1}, \mathbf{1})$, and $\left(l_{V}, m_{V}\right),\left(l_{\Sigma}, m_{\Sigma}\right),\left(l_{\Phi}, m_{\Phi}\right)$ and $\left(l_{\Phi^{c}}, m_{\Phi^{c}}\right)$ are equal to $(0,0) \otimes(1,1) \otimes(0,0)$ if the gauge fields were in the representation $(\mathbf{1}, \mathbf{3}, \mathbf{1})$, and $\left(l_{V}, m_{V}\right),\left(l_{\Sigma}, m_{\Sigma}\right),\left(l_{\Phi}, m_{\Phi}\right)$ and $\left(l_{\Phi^{c}}, m_{\Phi^{c}}\right)$ are equal to $(0,0) \otimes(0,0) \otimes(1,1)$ if the gauge fields were in the representation $(\mathbf{1}, \mathbf{1}, \mathbf{3})$, and $\left(l_{V}, m_{V}\right),\left(l_{\Sigma}, m_{\Sigma}\right),\left(l_{\Phi}, m_{\Phi}\right)$ and $\left(l_{\Phi^{c}}, m_{\Phi^{c}}\right)$ are equal to $(2,0) \otimes(1,0) \otimes$ $(1,0)$ if the gauge fields were in the representation $(\mathbf{6}, \mathbf{2}, \mathbf{2})$.

In order to have the models with $S U(3) \times S U(2) \times U(1)^{2}$ gauge symmetry and 4-dimensional $N=1$ supersymmetry for the zero modes, we obtain the following constraints on $n_{i}$

(a) $3 n_{1}+n_{2}=0 \bmod n$,

(b) $n_{3}+n_{4}=0 \bmod n$,

(c) $n_{1} \neq n_{2} \bmod n$,

(d) $n_{3} \neq n_{4} \bmod n$,

(e) $\left|n_{1}-n_{2}\right| \neq 1$ and $n-2 \bmod n$,

(f) $\left|n_{3}-n_{4}\right| \neq 1$ and $n-2 \bmod n$,

(g) $n_{i}+n_{j}+n_{k} \neq 0,1$ and $n-2 \bmod n$, for $i, j=1,2$ and $k=3,4$.

Because $R_{\Omega} \subset S U(4) \times S U(2)_{L} \times S U(2)_{R}$, we obtain the constraints (a) and (b). And the constraints (c) and (d) will break the $S U(4)$ down to $S U(3) \times U(1)$ and $S U(2)_{R}$ down to $U(1)$, respectively. In addition, the constraints (e) and (f) will project out all the zero modes of $\Sigma, \Phi$ and $\Phi^{c}$ in the representations $(\mathbf{1 5}, \mathbf{1}, \mathbf{1}),(\mathbf{1}, \mathbf{1}, \mathbf{3})$, and the constraint (g) will project out all the zero modes of $V, \Sigma, \Phi$ and $\Phi^{c}$ in the representation $(\mathbf{6}, \mathbf{2}, \mathbf{2})$. By the way, the zero modes of $\Sigma, \Phi$ and $\Phi^{c}$ in the representation $(\mathbf{1}, \mathbf{3}, \mathbf{1})$ are automatically projected out.

Let us give the model with $Z_{16}$ symmetry, the matrix representation for $R_{\Omega}$ is

$$
R_{\Omega}=\left(\omega^{5}, \omega^{5}, \omega^{5}, \omega\right) \otimes(+1,+1) \otimes\left(\omega^{3}, \omega^{13}\right) .
$$

It is easy to check that all the constraints are satisfied.

First, we consider that the extra space manifold is the annulus $A^{2}$. For the zero modes, we have 4-dimensional $N=1$ supersymmetry and $S U(3) \times S U(2) \times U(1)^{2}$ gauge symmetry in the bulk and on the 4-branes at $r=R_{1}$ and $r=R_{2}$. Including 
the KK states, we will have the 4-dimensional $N=4$ supersymmetry and $S O(10)$ gauge symmetry in the bulk, and on the 4-branes at $r=R_{1}$ and $r=R_{2}$.

Second, we consider that the extra space manifold is the disc $D^{2}$. For the zero modes, we have 4-dimensional $N=1$ supersymmetry and $S U(3) \times S U(2) \times U(1)^{2}$ gauge symmetry in the bulk and on the 4-brane at $r=R_{2}$. Including all the KK states, we will have the 4 -dimensional $N=4$ supersymmetry and $S O(10)$ gauge symmetry in the bulk, and on the 4-brane at $r=R_{2}$. Moreover, because the origin $(r=0)$ is the fixed point under the $Z_{16}$ symmetry, we always have the 4-dimensional $N=1$ supersymmetry and $S U(3) \times S U(2) \times U(1)^{2}$ gauge symmetry on the 3 -brane at origin in which only the zero modes exist.

\section{3 $S O(M)$ Breaking on $M^{4} \times A^{2}$ and $M^{4} \times D^{2}$}

On the space-time $M^{4} \times A^{2}$ and $M^{4} \times D^{2}$, we can break any 6-dimensional $N=2$ supersymmetric $S O(M)$ models down to the 4-dimensional $N=1$ supersymmetric $S U(3) \times S U(2) \times U(1)^{n-3}$ models where $n$ is the rank of the group $S O(M)$. The method is similar to above. We first decompose the $S O(M)$ group into the product of $S U\left(m_{i}\right)$ and/or $U(1)$ groups, then project out all the zero modes of the nonStandard Model like gauge fields and all chiral multiplets. For instance, $S O(12)$, we can decompose it into $S U(6) \times U(1)$ or $S U(4) \times S U(4)$, then discuss $S O(12)$ breaking by using above method and the branching rules for various representations in Ref. [8].

\section{$5 \quad E_{8}$ Breaking on $M^{4} \times A^{2}$ and $M^{4} \times D^{2}$}

Although from the phenomenological point of view $E_{8}$ is not interesting, we will discuss the $E_{8}$ breaking on the space-time $M^{4} \times A^{2}$ and $M^{4} \times D^{2}$ because of technical interesting. We will show how to break the 6-dimensional $N=2$ supersymmetric $E_{8}$ model down to 4-dimensional $N=1$ supersymmetric $S U(3) \times S U(2) \times U(1)^{4}$ model for the zero modes. By the way, we would like to point out that on the 6-dimensional space-time $M^{4} \times S^{1} /\left(Z_{2} \times Z_{2}^{\prime}\right) \times S^{1} /\left(Z_{2} \times Z_{2}^{\prime}\right)$, we can break the 6 -dimensional $N=2$ supersymmetric $E_{8}$ model down to 4-dimensional $N=1$ supersymmetric $S O(10) \times$ $U(1)$ model, or $S U(3) \times S U(3) \times S U(3) \times S U(2) \times U(1)$ model, etc, however, we can not break it down to the 4-dimensional $N=1$ supersymmetric $S U(3) \times S U(2) \times$ $U(1)^{4}$ model for the zero modes unless we consider the 8-dimensional space-time $M^{4} \times S^{1} /\left(Z_{2} \times Z_{2}^{\prime}\right) \times S^{1} /\left(Z_{2} \times Z_{2}^{\prime}\right) \times S^{1} /\left(Z_{2} \times Z_{2}^{\prime}\right) \times S^{1} /\left(Z_{2} \times Z_{2}^{\prime}\right)$.

$E_{8}$ has following maximal subgroups: $S U(3) \times E_{6}, S U(4) \times S O(10), S U(5) \times$ $S U(5), S U(2) \times E_{7}, S O(16)$ and $S U(9)$ [8]. And the first three maximal subgroups are interesting in the weakly coupled heterotic $E_{8} \times E_{8}$ string theory or M-theory on $S^{1} / Z_{2}$ model buildings, so, we decompose the gauge fields according to the first three maximal subgroups. As we know, the gauge fields of $E_{8}$ are in the adjoint representation with dimension 248, under the maximal subgroup $S U(3) \times E_{6}$, the gauge fields decompose as [8]

$$
248=(\mathbf{8}, \mathbf{1}) \oplus(\mathbf{1}, \mathbf{7 8}) \oplus(\mathbf{3}, \mathbf{2 7}) \oplus(\overline{\mathbf{3}}, \overline{\mathbf{2 7}}),
$$


and under the maximal subgroup $S U(4) \times S O(10)$, the gauge fields decompose as

$$
248=(\mathbf{1 5}, \mathbf{1}) \oplus(\mathbf{1}, 45) \oplus(\mathbf{4}, \mathbf{1 6}) \oplus(\overline{\mathbf{4}}, \overline{\mathbf{1 6}}) \oplus(\mathbf{6}, \mathbf{1 0})
$$

and under the maximal subgroup $S U(5) \times S U(5)$, the gauge fields decompose as 8

$$
248=(\mathbf{2 4}, \mathbf{1}) \oplus(\mathbf{1}, \mathbf{2 4}) \oplus(\mathbf{5}, \mathbf{1 0}) \oplus(\overline{\mathbf{5}}, \overline{\mathbf{1 0}}) \oplus(\mathbf{1 0}, \overline{\mathbf{5}}) \oplus(\overline{\mathbf{1 0}}, \mathbf{5})
$$

In this paper, we only discuss the $E_{8}$ breaking by decomposing $E_{8}$ into $S U(5) \times S U(5)$. Similarly, noticing that the $E_{6}$ breaking has been discussed in Ref. [4] and $S O(10)$ breaking has been discussed in the last section, and using the branching rules for various representations in Ref. [B], one can discuss the $E_{8}$ breaking by decomposing $E_{8}$ into $S U(3) \times E_{6}$ or $S U(4) \times S O(10)$.

Assume that we have $Z_{2 n}$ symmetry on $A^{2}$ or $D^{2}$ and $\Omega$ is a generator of $\Gamma=Z_{2 n}$, we choose the following matrix representation for $\Omega$, which will give us the representations of all the elements in $\Gamma$,

$$
R_{\Omega}=\left(+1,+1,+1, \omega^{n}, \omega^{n}\right) \otimes\left(\omega^{n_{1}}, \omega^{n_{2}}, \omega^{n_{3}}, \omega^{n_{4}}, \omega^{n_{5}}\right),
$$

where $\omega=e^{\mathrm{i} \frac{2 \pi}{2 n}}$. Moreover, $\left(l_{V}, m_{V}\right),\left(l_{\Sigma}, m_{\Sigma}\right),\left(l_{\Phi}, m_{\Phi}\right)$ and $\left(l_{\Phi^{c}}, m_{\Phi^{c}}\right)$ are equal to $(1,1) \otimes(0,0)$ if the gauge fields were in the representation $(\mathbf{2 4}, \mathbf{1})$, and $\left(l_{V}, m_{V}\right)$, $\left(l_{\Sigma}, m_{\Sigma}\right),\left(l_{\Phi}, m_{\Phi}\right)$ and $\left(l_{\Phi^{c}}, m_{\Phi^{c}}\right)$ are equal to $(0,0) \otimes(1,1)$ if the gauge fields were in the representation $(\mathbf{1}, \mathbf{2 4})$, and $\left(l_{V}, m_{V}\right),\left(l_{\Sigma}, m_{\Sigma}\right),\left(l_{\Phi}, m_{\Phi}\right)$ and $\left(l_{\Phi^{c}}, m_{\Phi^{c}}\right)$ are equal to $(1,0) \otimes(2,0)$ if the gauge fields were in the representation $(\mathbf{5}, \mathbf{1 0})$, and $\left(l_{V}, m_{V}\right)$, $\left(l_{\Sigma}, m_{\Sigma}\right),\left(l_{\Phi}, m_{\Phi}\right)$ and $\left(l_{\Phi^{c}}, m_{\Phi^{c}}\right)$ are equal to $(0,1) \otimes(0,2)$ if the gauge fields were in the representation $(\overline{\mathbf{5}}, \overline{\mathbf{1 0}})$, and $\left(l_{V}, m_{V}\right),\left(l_{\Sigma}, m_{\Sigma}\right),\left(l_{\Phi}, m_{\Phi}\right)$ and $\left(l_{\Phi^{c}}, m_{\Phi^{c}}\right)$ are equal to $(2,0) \otimes(0,1)$ if the gauge fields were in the representation $(\mathbf{1 0}, \overline{\mathbf{5}})$, and $\left(l_{V}, m_{V}\right)$, $\left(l_{\Sigma}, m_{\Sigma}\right),\left(l_{\Phi}, m_{\Phi}\right)$ and $\left(l_{\Phi^{c}}, m_{\Phi^{c}}\right)$ are equal to $(0,2) \otimes(1,0)$ if the gauge fields were in the representation $(\overline{\mathbf{1 0}}, \mathbf{5})$,

In order to have the models with $S U(3) \times S U(2) \times U(1)^{4}$ gauge symmetry and 4-dimensional $N=1$ supersymmetry for the zero modes, we obtain the following constraints on $n_{i}$

$$
\text { (a) } n_{1}+n_{2}+n_{3}+n_{4}+n_{5}=0 \bmod 2 n \text {, }
$$

(b) $n_{i} \neq n_{j} \bmod 2 n$, for $i, j=1,2,3,4,5$ and $i \neq j$,

(c) $\left|n_{i}-n_{j}\right| \neq 1$ and $n-2 \bmod 2 n$, for $i, j=1,2,3,4,5$ and $i \neq j$,

(d) $\left|n_{i}\right| \neq 0,1$ and $n-2 \bmod 2 n$, for $i=1,2,3,4,5$,

(e) $\left|n-n_{i}\right| \neq 0,1$ and $n-2 \bmod 2 n$, for $i=1,2,3,4,5$, 


$$
\begin{aligned}
& \text { (f) }\left|n_{i}+n_{j}\right| \neq 0,1 \text { and } n-2 \bmod 2 n \text {, for } i, j=1,2,3,4,5 \text {, } \\
& \text { (g) }\left|n_{i}+n_{j}+n\right| \neq 0,1 \text { and } n-2 \bmod 2 n \text {, for } i, j=1,2,3,4,5 \text {. }
\end{aligned}
$$

Because $R_{\Omega} \subset S U(5) \times S U(5)$, we obtain the constraint (a). And the constraint (b) will break the second $S U(5)$ down to $U(1)^{4}$. In addition, the constraint (c) will project out all the zero modes of $\Sigma, \Phi$ and $\Phi^{c}$ in the representation $(\mathbf{1}, \mathbf{2 4})$, and the constraints (d), (e), (f) and (g) will project out all the zero modes of $V, \Sigma, \Phi$ and $\Phi^{c}$ in the representations $(\mathbf{5}, \mathbf{1 0}),(\overline{\mathbf{5}}, \overline{\mathbf{1 0}}),(\mathbf{1 0}, \overline{\mathbf{5}})$ and $(\overline{\mathbf{1 0}}, \mathbf{5})$. By the way, the zero modes of $\Sigma, \Phi$ and $\Phi^{c}$ in the representation $(\mathbf{2 4}, \mathbf{1})$ are automatically projected out if $n>2$.

Let us give the model with $Z_{66}$ symmetry, the matrix representation for $R_{\Omega}$ is

$$
R_{\Omega}=(+1,+1,+1,-1,-1) \otimes\left(\omega^{3}, \omega^{6}, \omega^{9}, \omega^{12}, \omega^{36}\right) .
$$

It is easy to check that all the constraints are satisfied.

First, we consider that the extra space manifold is the annulus $A^{2}$. For the zero modes, we have 4-dimensional $N=1$ supersymmetry and $S U(3) \times S U(2) \times U(1)^{4}$ gauge symmetry in the bulk and on the 4-branes at $r=R_{1}$ and $r=R_{2}$. Including the KK states, we will have the 4-dimensional $N=4$ supersymmetry and $E_{8}$ gauge symmetry in the bulk, and on the 4-branes at $r=R_{1}$ and $r=R_{2}$.

Second, we consider that the extra space manifold is the disc $D^{2}$. For the zero modes, we have 4-dimensional $N=1$ supersymmetry and $S U(3) \times S U(2) \times U(1)^{4}$ gauge symmetry in the bulk and on the 4 -brane at $r=R_{2}$. Including all the KK states, we will have the 4 -dimensional $N=4$ supersymmetry and $E_{8}$ gauge symmetry in the bulk, and on the 4 -brane at $r=R_{2}$. In addition, because the origin $(r=0)$ is the fixed point under the $Z_{66}$ symmetry, we always have the 4-dimensional $N=1$ supersymmetry and $S U(3) \times S U(2) \times U(1)^{4}$ gauge symmetry on the 3 -brane at origin in which only the zero modes exist. And if we put the Standard Model fermions on the 3-brane at origin, the extra dimensions can be large and the gauge hierarchy problem can be solved for there does not exist the proton decay problem at all.

\section{Remarks, Possible Questions and Generalization}

First, we would like to comment on the one-loop effective potential. It was pointed out that the representations $\left(R_{\Gamma_{I}}\right)$ of the discrete symmetries $\left(\Gamma_{I}\right)$ are not arbitrary parameters of the theory and are determined dynamically [6]. The tree-level effective potential is trivial, so, we need to consider the one-loop effective potential which can be done by the well-known results of the background field gauge. However, if there exist non-broken supersymmetry at the compactification scale, it is not difficult for us to show that the one-loop contribution is zero. Therefore, the 4-dimensional $N=1$ supersymmetry breaking scale must be much lower than the compactification scale, which is true for the supersymmetric GUT models in Ref. [1, 2, 6, ㄴ, where 
the 4-dimensional $N=1$ supersymmetry breaking scale is around $\mathrm{TeV}$, and the compactification scale or GUT scale is around $10^{16} \mathrm{GeV}$. This also implies that if we considered the non-supersymmetric GUT breaking by discrete symmetry, we must calculate the one-loop effective potential and determine the representations $\left(R_{\Gamma_{I}}\right)$ of the discrete symmetries $\left(\Gamma_{I}\right)$ dynamically, $i$. e., we can not choose $R_{\Gamma_{I}}$ arbitrarily.

However, there might still exist the question in the discrete symmetry approach. Because the vacua are degenerate, why the nature chooses the Standard Model like gauge symmetry as vaccum. For example, we consider the supersymmetric $S U(5)$ model, the $S U(5)$ gauge symmetry can be unbroken, or broken down to the $S U(4) \times U(1)$ or $S U(3) \times S U(2) \times U(1)$ gauge symmetry, the question is why the $S U(5)$ Model is broken down to the Standard Model.

Second, when we consider the 6-dimensional supersymmetric GUT breaking, we often consider the 6-dimensional $N=2$ supersymmetry in order to avoid the gauge anomaly. Because we can not have the hypermultiplets in the bulk, we have to put the Standard Model fermions and Higgs doublets on the fixed point or line. So, we need to understand how to localize the matter fields and Higgs fields at the fixed point or line.

Third, we can not reduce the rank of GUT group in the discrete symmetry approach because the cyclic group is abelian. If we want to reduce the rank of the GUT group, we might need to consider the continuous symmetry on the extra space manifold. It is also interesting to generalize the discrete symmetry approach on the non-commutative extra dimensions.

\section{Conclusion}

We study the principles of the gauge symmetry and supersymmetry breaking due to the local or global discrete symmetries on the extra space manifold. We show that the gauge symmetry breaking by Wilson line is the special case of the discrete symmetry approach where all the discrete symmetries are global and act freely on the extra space manifold. As applications, we discuss the $N=2$ supersymmetric $S O(10)$ and $E_{8}$ breaking on the space-time $M^{4} \times A^{2}$ and $M^{4} \times D^{2}$, and point out that similarly one can study any $N=2$ supersymmetric $S O(M)$ breaking. We also comment on the one-loop effective potential, the possible questions and generalization.

\section{Acknowledgments}

This work was supported in part by the U.S. Department of Energy under Grant No. DOE-EY-76-02-3071. 


\section{References}

[1] Y. Kawamura, Prog. Theor. Phys. 103 (2000) 613 hep-ph/9902423, hepph/0012125, hep-ph/0012352; G. Altarelli and F. Feruglio, hep-ph/0102301; L. Hall and Y. Nomura, hep-ph/0103125; T. Kawamoto and Y. Kawamura, hep-ph/0106163; A. Hebecker and J. March-Russell, hep-ph/0106166, hepph/0107039; R. Barbieri, L. Hall and Y. Nomura, hep-ph/0106190, hepth/0107004; A. B. Kobakhidze, hep-ph/0102323; T. Li, hep-th/0107136, hepph/0108120; J. A. Bagger, F. Feruglio and F. Zwirner, hep-th/0107128; A. Masiero, C. A. Scrucca, M. Serone and L. Silvestrini, hep-ph/0107201; L. Hall, H. Murayama and Y. Nomura, hep-th/0107245; C. Csaki, G. D. Kribs and J. Terning, hep-ph/0107266; L. Hall, Y. Nomura and D. Smith, hep-ph/0107331; N. Maru, hep-ph/0108002; T. Asaka, W. Buchmuller and L. Covi, hepph/0108021; L. Hall, Y. Nomura, T. Okui and D. Smith, hep-ph/0108071; N. Haba, T. Kondo, Y. Shimizu, T. Suzuki and K. Ukai, hep-ph/0108003; L. Hall, J. March-Russell, T. Okui and D. Smith, hep-ph/0108161; R. Dermisek and A. Mafi, hep-ph/0108139; T. Watari and T. Yanagida, hep-ph/0108152; Y. Nomura, hep-ph/0108170; Q. Shafi and Z. Tavartkiladze, hep-ph/0108247; L. Hall, J. March-Russell, T. Okui and D. Smith, hep-ph/0108161; N. Haba, hep-ph/0110164; R. Barbieri, L. Hall and Y. Nomura, hep-ph/0110102; L. Hall and Y. Nomura, hep-ph/0111068; M. Kubo, C. S. Lim, H. Yamashita, hepph/0111327; G. Bhattacharyya and K. Sridhar, hep-ph/0111345; H. D. Kim, J. E. Kim, H. M. Lee, hep-ph/0112094; N. Haba, T. Kondo, Y. Shimizu, hepph/0112132; A. Hebecker, hep-ph/0112230.

[2] T. Li, hep-ph/0108238.

[3] T. Li, hep-th/0110065.

[4] C. S. Huang, J. Jiang, T. Li and W. Liao, hep-th/0112046.

[5] Y. Hosotani, Phys. Lett. B126 (1983) 309; Phys. Lett. B129 (1983) 193; E. Witten, Nucl. Phys. B258 (1985) 75; J. D. Breit, B. A. Ovrut and G. C. Segre, Phys. Lett. B158 (1985) 33.

[6] Y. Hosotani, Annals of Physics 190 (1989) 233.

[7] N. Arkani-Hamed, T. Gregoire and J. Wacker, hep-th/0101233.

[8] R. Slansky, Phys. Rept. 79 (1981) 1. 